\title{
The Design and Implementation of the Tracking and Monitoring System for Gerocomium based on Semi-active RFID Technology
}

\author{
Xiaoyong Zhong, Zhangbing Li \\ School of Computer Science and Engineering \\ Hunan University of Science and Technology \\ Xiangtan, Hunan, 411201, P.R.China \\ E-mail: Lzb_xt@163.com
}

\author{
Yuzhen Liu, Zilan Zhu \\ School of Computer Science and Engineering \\ Hunan University of Science and Technology \\ Xiangtan, Hunan, 411201, P.R.China \\ E-mail: 21182240@qq.com
}

\begin{abstract}
As the ageing of population is getting worse in China, both people and society pay more attention to the support and nursing of the elderly, establishing many gerocomium and monitoring system for the elderly. The monitoring system mainly focuses on the real-time monitoring, however, it is still not accurate and promptly enough in aspects of nursing, quick response, and fixed position accuracy. In order to reduce nursing accidents of the elderly, the semi-active radio frequency identification (RFID) technology has been utilized in the monitoring and positioning system. The tracking and monitoring system of gerocomium has been built based on the idea of the Internet of Things Engineering, realizing the function of quick fixed position, active help-seeking, basic information management, and information processing of activities of the elderly. Therefore, in the real-time monitoring system, problems like inaccurate positioning, slow response, and delay of nursing and assisting the elderly are properly solved.
\end{abstract}

Keywords-semi-active RFID; gerocomium; Internet of Things; tracking; monitoring; active assistance

\section{INTRODUCTION}

With the improvement of living standards and health conditions, the ageing of the population has become a major urgently unsolved problem by many countries. In China, the number of the aging population grows rapidly. The sixth national census data in 2010 shows that, elderly population aged over 60 occupies 13.26 percent of the country's total population, and the population over 65 occupies 8.87 percent of the total population. It is predictable that the aging population will reach 200 million in 2014 and 300 million in $2026^{[1]}$.

The problem of the aging population's support and endowment is a major social and strategic issues related to the sustainable development of the country and national welfare and the people's livelihood. Certainly, the gerocomium is the best choice to solve this social problem. And the utilization of modern advanced technology is of great significance for improving gerocomium's service system, which is an urgent need to establish a sound pension system.

The management system of traditional gerocomium is using a mere manual mode, which wastes a lot of human resources and reduces the security and reliability. Now some gerocomiums use the traditional tracking and monitoring technology like video, wireless. Although it improves the efficiency of the monitoring and management of elderly people's activities to some extent, there still exists the problem of untimely positioning. In the thesis, it points out that the Semi-active RFID technology is utilized in the monitoring and positioning system, establishing a gerocomium tracking and monitoring system based on the idea of the Internet of Things Engineering ${ }^{[2]}$. For the elderly, this system can provide real-time monitoring, accurate positioning, quick response, timely nursing treatment, and activities' record, and it also has the function of active helpseeking. Also, this system provides effective solutions to improve service levels of gerocomium, and has competitive advantages of low-cost and practical application prospects.

\section{AN ANALYSIS OF SYSTEM REQUIREMENTS}

Generally, the structure of the gerocomium consists of square, playground, canteens, place of entertainment, and residential buildings. This system not only tracks and monitors outdoor activities of the elderly but also their indoor activities, because of the large area of the gerocomium; administrators can not give consideration to every old man in every place. When there is an emergency of the elderly, the system should implement the function of active help-seeking by calling, thus, the nursing staff and manager can locate the position of the old man in trouble as soon as possible, and give first aid for him. The general facilities of gerocomium are shown in Fig.1.

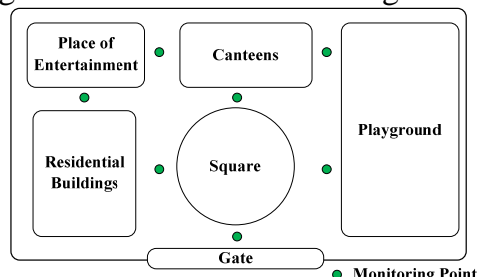

Figure 1. The general facilities of gerocomium

The Global Positioning System (GPS) that based on Satellite Communications is insufficient in the positioning precision of indoor complex environment ${ }^{[3]}$. While Zigbee, Wifi wireless communication can not meet the demands of the tracking and monitoring system for gerocomium because of its complex requirements of client, object identification, high cost and energy consumption. Considering the aspects 
of actual cost, positioning accuracy, object identification, and energy consumption, the RFID technology, as the main promoter of the Internet of things' application, carrying on non-contact radio frequency function type bidirectional data communication exchange to achieve recognition and positioning has the advantages of low cost, large transmission range, non-contact and non-line-of-sight ${ }^{[4]}$. Obviously, the RFID technology wins the competitive edge on the precise positioning of short distance compared with GPS, Zigbee, WiFi and infrared, ultrasonic positioning technology. And it meets the needs in flexible arrangement of monitoring site, high accuracy and short distance, and data record.

According to different supply modes of the energy requirement, RFID tags can be divided into active, passive, and semi-active tag ${ }^{[5]}$. Currently, the active RFID tags' weak points lie in uncertain reading distance and tag's short service life, while the passive RFID tags only can work within the reader's reading range but not send information actively, therefore can not content the needs of the elderly to call for help. And the passive RFID tags are easily absorbed by the body and obstructed by the metal material which can not be put together with it. The semi-active RFID tags are usually in a dormant state, and they can transmit information only after the activation of the low-frequency locator. Therefore, it can prolong the active RFID tag more than double the service life, and the activation range of the locator can easily identify the active RFID tag's position. The existing reader's technology is just available to handle the active tag data within dozens of meters regardless whether the data is necessary or not, hence, the effectiveness of the data is low. But the semi-active technology helps the reader only to deal with the tags within the locator's range, which makes all the electronic tag datas are effective and strengthens the efficiency of data. Correspondingly, the number of tags that the reader can read simultaneously is increased $^{[6]}$.

From the demand of indoor and outdoor positioning, system implementation cost and system functional requirements, semi-active RFID technology is introduced to locating and monitoring system and the gerocomium's tracking and monitoring system is established based on the idea of the Internet of Things Engineering. Through this system, following functions will be implemented:

- Basic information management: to add, delete, modify, and query the information of the elderly and the staff

- Positioning management: to implement the real-time query of the position of the personnel who has RFID tag, or query past position of the personnel who has RFID tag for a time.

- Activities' trajectory reproduction:to show historic trajectory of personnel who has RFID tag through activities list or electronic map.

- Call and response: when the elderly has difficulties and need help, the system implement the function call for help through pressing the button, then the system will show the information of the help: the elderly's name and the elderly's position, etc.

- Low pressure alarm: the service life of normal use of the semi-active RFID tags up to 2 to 3 years, and battery was changed easily. When RFID tags are lack of electricity, the system will automatically provides the low pressure alarm.

\section{SYSTEM DESIGN}

\section{A. The Relevant Principles}

- the principle of personnel positioning: Locators that installed in different areas have their unique address code, When people with the RFID tag comes into the locator activation range, the RFID tag is activated and works normally, sending wirelessly outwards both tag ID number and the address code of locator that activate tag. Also, the uploaded locator address code will change along with the tag moves from one locator to another. As a result, through the locator's address code, the position of the tag can be pinpointed. The wireless information sent by the tag is received by a reader. Then, the reader, through the switchboard in the wired way, will upload information to the monitoring server of the management center for processing.

- The principle of the elderly's active help-seeking: When the elderly needs help, he can press the call button of the wristband at anywhere in the gerocomium, RFID tags will send information that is different from the usual card number to reader, which on behalf of the abnormal state. Reader transmits this information to the central monitoring server-side through wired-link, and application software will achieve the functionality of the current card's call for help.

- The principles of solving the problem of Semi-active RFID signal collision: The RFID wristband that was actived by low-frequency locator send the information which strap with the address-code of the locator to reader, which can determine the position of the elderly easily. But the information that the elderly send out through the Semi-active RFID button can be received by several reader within the effective distance, and it can generate reader collision, so that the system can't distinguish the location of this tag. In this thesis, the system uses the method of signal strength detection. When the RFID tag is to move to the overlap area of the two readers, and the intensity of a signal obtained by the two readers is inconsistent, the contrast signal strength determines a specific location of the RFID tag ${ }^{[7]}$.

\section{B. System Architecture Design}

The tracking and monitoring system of gerocomium is made up of the semi-active RFID tags, the low-frequency 
locator, reader, network equipment, computer, server and system software. System Architecture shown in Fig.2:

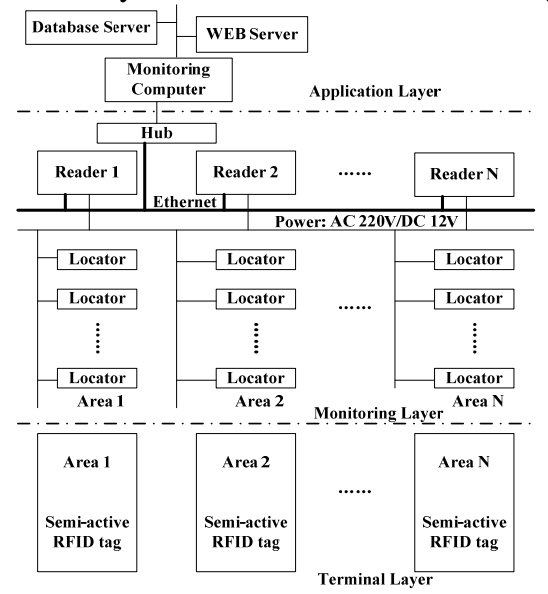

Figure 2. System architecture

In Fig.2, system equipment's terminal layer uses the semi-active RFID wristbands which are dual-frequency tags $(125 \mathrm{kHz}+433 \mathrm{MHz})$, and the operating mode of wristbands is that it uses the high-frequency $(433 \mathrm{MHz})$ send data after it has been activated by low-frequency $(125 \mathrm{kHz})$ trigger or the elderly actively key. The low-frequency locator in the monitoring layer send low frequency $(125 \mathrm{kHz})$ trigger at regular intervals, which helps to activate the semi-active RFID wristbands in its range. The reader has been in a passive state, mainly receiving data from the tags and transmitting the information to monitor server-side in time. Monitoring computer in the application layer is mainly used to filter and format the data from the reader in order to provide this data to other applications on the server; the system uses B/S model, and web server and database server are set up in the Administration Center. So the relatives of the elderly can know the situation of the elderly at any time by a computer or mobile phone through logging in the Management Center WEB server.

\section{System Monitoring Design}

Considering a wider area of gerocomium, the design of the outdoor positioning helps to inspect the area at which the elderly states. Additionally, an indoor positioning is convenient for locate the elderly more specifically (such as a room). Therefore, the design of data-monitoring-points (DMP) is the key point of the entire system, and it is used to collect data and get the time and location information of staff and it is the basis for other applications. Therefore, the installation of DMP should be taken full account of the actual situation of the gerocomium. And the monitoring area should be divided reasonably, and a large number of locators are required in the areas where many people frequent, and the entire gerocomium should be covered a certain number of readers without blind spots. The deployment of the DMP of the gerocomium is shown in Fig.1.

\section{System Database Design}

Through analyzing the requirement of information management, the focuses of monitoring and management lie in indoor and outdoor positioning and basic information management for the elderly and staff. It is important to abstract the entity of the region of the gerocomium, and it is necessary to identify the elderly, nursing person, and administrators, recording the information of time and location.

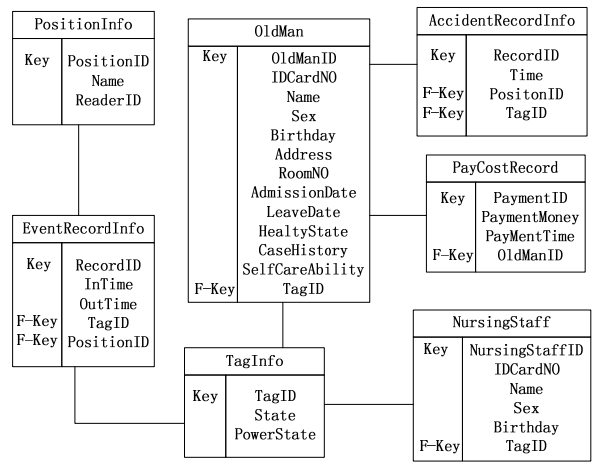

Figure 3. E-R diagram of system information

Fig. 3 shows the ER diagram about the elderly entity, in a central location, there is an entity of the elderly named Oldman, and its main properties include name, identity card number, the elderly number, address, date of birth, admission time , discharge from hospital, medical history, current health status, self-care ability and so on, and PositionInfo is the regional location information of gerocomium, while AccidentRecordInfo is the information associated with the accident record of the elderly. Besides, the data files are stored in the monitoring server-side of the Management Center.

\section{THE IMPLEMENTATION OF SYSTEM}

\section{A. The Construction of Platform}

The tracking and monitoring system of the gerocomium can be formed by the semi-active RFID tags, the lowfrequency locator, the reader, the network equipment, computer, the server and system software. Application server is a management center based on the Windows XP platform B/S model and developed by PHP + MYSQL5 technology programming ${ }^{[8-9]}$. This system can process the real-time data that collected by the reader and it also can display the location information of the personnel in the management center on the electronic map. The hardware configuration of the system is shown in TABLE I:

TABLE I. HARDWARE DEVICES

\begin{tabular}{|c|c|c|}
\hline Name & Numbers & Distance \\
\hline Locator & 62 & $1 \sim 5 \mathrm{~m}$ \\
\hline Reader & 13 & $100 \mathrm{~m}$ \\
\hline Semi-active RFID Wristbands & 96 & $100 \mathrm{~m}$ \\
\hline Computer & 1 & - \\
\hline
\end{tabular}


In experimental units of the gerocomium, like the cafeteria's entrance, the low-frequency locator and reader are required to be installed in appropriate location. When people pass by, the semi-active RFID wristband is activated, sending out its own ID and locator address code to the reader, by the address code information, the system can confirm that people's entrance to the area and display on the electronic map.

Inside a building, taking the residential building as an example, Each room, stairs' passage of each floor, and the elevator should be installed one locator, respectively. And there should be two readers in every floor ${ }^{[10-11]}$. By doing so, the specific location of the elderly (accurate to the room) can be inspected at any time. If there is someone has an accident or in trouble, the nursing administrators will arrive at the scene as quick as possibe, greatly reducing the probability of turning accidents into worse situations.

\section{B. Experimental Results and Analysis}

Through the implementation of the system in gerocomium, the following functions have been realized. There are personnel positioning, timely response to the elderly's call for help by pushing alarm button, the elderly historic trajectory queries and basic information management. During the test, 96 cards are given out in experimental units of the gerocomium, carrying out a test lasting twenty days. Statistical analysis of the data in the database is obtained from the test results that are shown in TABLE II:

TABLE II. TEST RESULTS

\begin{tabular}{|c|c|c|c|c|c|}
\hline & Events & Accidents & Mistake & Errors & Total \\
\hline Times & 17831 & 2028 & 54 & 8 & 19921 \\
\hline Ratio & $89.51 \%$ & $10.18 \%$ & $0.27 \%$ & $0.04 \%$ & $100 \%$ \\
\hline
\end{tabular}

The test results prove that: tracking and monitoring system in the gerocomium works in stable condition, a total of 19,921 times reader, the average misreading rate was $0.27 \%$, misjudgment was $0.04 \%$, achieved good results in personnel positioning and alarm event handling functionality. effective solve the fast positioning of the elderly, real-time monitoring, responding and caring and other issues which is not timely.

\section{CONCLUSION}

The future belongs to the Internet of Things. Through the introduction of the RFID technology, the system is employed in the gerocomium to monitor and track the daily life of the elderly, which is in accord with the service direction of the pension for the aged in the future, and the system is also a solution of the ageing of population. It has a beneficial effect on the perfection of the endowment insurance and economic development. The tracking and monitoring system based on the semi-active RFID technology in geracomium solves many intractable problems of the real-time monitoring system. With this new system, accurate fixed position and timely response to the active help-seeking and nursing treatment are easily achievable. In addition, this system is in charge of the information of activities and nursing for the elderly, which heightens the security and administrative effectiveness of the geracomium. To conclude, this system is of great significance in practice. It belongs to the application program of the Internet of things, and it is an important solution to the ageing of population. Moreover, this system can be utilized for institutions of nursing children or other vulnerable groups, and it will be popularized and widespread in the future.

\section{ACKNOWLEDGMENT}

This paper is supported by NSFC, under grant number 60673119, and by Scientific Research Fund of Hunan Province Office of Sci. \& Tec., under grant number: B11219, Scientific Research Fund of Hunan Province Office of Education, under grant number: 11C0533.

\section{REFERENCES}

[1] Xu X, "Problems of Endowment Insurance System Under the Aging Population Background in China,” Economic Research Guide, 2012, NO.08, pp.101-102.

[2] Wu G Y, "The wisdom of the Internet of things: perception of the technology in China and the world," Beijing: Mechanical Industry Press, 2010,pp.1-70.

[3] Lin M, Wang G, "Study on the positioning of RFID Tags," The Thesis of Jiangsu University, 2007.

[4] Klaus Finkenzeller, "Radio frequency identification technology," Beijing:Electronic Industry Press, 2006,pp.15-60.

[5] Shan C G, "Principles and Applications of Radio Frequency Identification (RFID),” Beijing:Electronic Industry Press, 2008, pp.30-120.

[6] Zhang J G, Xu R, Wang D Z, "Research on Wireless Attendence System for Home-school Interaction Based on RFID Technology. 2011, NO.10, pp.38-40.

[7] Ni S P, Deng L H, Xiang Y, "Design on the Personal Positioning System in the Well Based on RFID," Sci-Tech Information Development \& Economy, 2007, NO.25, doi: 1005-6033(2007)250210-02.

[8] Yong Fu, Li Jin, "PHP framework for advanced programming," Beijing: Tsinghua University Press, 2012, pp.27-464.

[9] Minghan Tang, "MySQL database development, optimization, and management and maintenance to layman,” Beijing: People's Posts and Telecommunications Press, 2008, pp.25-302.

[10] Joseph C, Chen Thomas J, Collins, "Creation of RFID Based Real Time Tracking (R-RTT) System for Small Healthcare Clinics.Journal Medical Systems,” December 2012, Volume36, pp.3851-3860.

[11] Xie Z J, "RFID and Sensor Networks: Architectures, Protocols, Security and Integrations,” Beijing: Mechanical Industry Press, 2012, pp.456-471. 